\title{
On the Existence and Uniqueness of the Solution of Linear Fractional Differential-Algebraic System
}

\author{
Zaiyong Feng ${ }^{1,2}$ and Ning Chen ${ }^{1}$ \\ ${ }^{1}$ College of Mechanical and Electronic Engineering, Nanjing Forestry University, Nanjing, Jiangsu 210037, China \\ ${ }^{2}$ Department of Social Sciences, Nanjing Institute of Railway Technology, Nanjing, Jiangsu 210031, China \\ Correspondence should be addressed to Ning Chen; chenning@njfu.com.cn
}

Received 26 December 2015; Accepted 7 March 2016

Academic Editor: Rosana Rodriguez-Lopez

Copyright (c) 2016 Z. Feng and N. Chen. This is an open access article distributed under the Creative Commons Attribution License, which permits unrestricted use, distribution, and reproduction in any medium, provided the original work is properly cited.

\begin{abstract}
The existence and uniqueness of the solution of a new kind of system-linear fractional differential-algebraic equations (LFDAE) are investigated. Fractional derivatives involved are under the Caputo definition. By using the tool of matrix pair, the LFDAE in which coefficients matrices are both square matrices have unique solution under the condition that coefficients matrices make up a regular matrix pair. With the help of equivalent transformation and Kronecker canonical form of the coefficients matrices, the sufficient condition for existence and uniqueness of the solution of the LFDAE in which coefficients matrices are both not square matrices is proposed later. Two examples are given to justify the obtained theorems in the end.
\end{abstract}

\section{Introduction}

The dynamical behavior of a mechanical system was usually modeled via differential-algebraic equations (DAE) whose general form appears as $F(t, y, \dot{y})=0$, including both differential and algebraic equations to describe the corresponding constraints, for example, by Newton's laws of motion or by position constraints such as the movement on a given surface. On the other hand, researchers had effectively solved engineering problems with fractional differential equations (FDE), which involves fractional derivatives $y^{(\alpha)}$ in the model [1-6].

Recently, some investigators tried using fractional differential-algebraic equations (FDAE), which denote the combination of DAE and FDE, in dealing with the studied system. The general form of FDAE appears as $F\left(t, y, y^{(\alpha)}\right)=0$, where $F, y, y^{(\alpha)}$ could be vectors if necessary. Till now, the majority of these attempts concentrated on the algorithms for solving the FDAE [7-10], while fundamental problems such as the existence and uniqueness of the solution were neglected.

Existence and uniqueness of the solution of the model are of great significance, since the existence of the solution guarantees the practicability of the model, while the uniqueness of the solution guarantees the validity of the obtained solution.
In this paper, the existence and uniqueness of the solution of linear fractional differential-algebraic systems are discussed to lay the groundwork for the further studies and applications.

\section{Linear Fractional \\ Differential-Algebraic System}

Consider the initial value problem for linear fractional differential-algebraic equations (LFDAE) in the form

$$
\begin{aligned}
E_{t_{0}} D_{t}^{(\alpha)} \mathbf{y} & =A \mathbf{y}+\mathbf{z}, \\
\left.\mathbf{y}\right|_{t=t_{0}} & =\mathbf{0},
\end{aligned}
$$

where $0<\alpha<1, \mathbf{y}(t) \in \mathbb{R}^{n}, \mathbf{z}(t) \in \mathbb{R}^{m}, \mathbf{y}(t), \mathbf{z}(t) \in$ $L_{1}(0, T), 0<t<T<+\infty, m \leq n . E, A \in R^{m \times n}$ are constant coefficients matrices. $t_{0} D_{t}^{(\alpha)}$ denotes Caputo's fractional derivative operator [11-13], $t_{0} D_{t}^{(\alpha)} y=(1 / \Gamma(1-$ $\alpha)) \int_{t_{0}}^{t}\left(y^{\prime}(\xi) /(t-\xi)^{\alpha}\right) d \xi$, since Caputo's fractional derivative allows us to couple the fractional differential equations with initial conditions in the traditional form $\left.\mathbf{y}\right|_{t=t_{0}}=\mathbf{y}_{\mathbf{0}}(=\mathbf{0})$. And it is linked to other fractional definitions under certain 
conditions [11]. For convenience, we write system (1) as $(E, A)$ in short.

For given $\mathbf{z}(t) \in \mathbb{R}^{m}$, whether there exists unique solution $\mathbf{y}(t) \in \mathbb{R}^{n}$ for LFDAE (1) is a problem of great significance in application and it is our main concern as well.

System (1) is also valid when $\left.\mathbf{y}\right|_{t=t_{0}} \neq \mathbf{0}$. In this case, by a simple coordinate transformation, we can obtain $E_{t_{0}} D_{t}^{(\alpha)} \mathbf{y}_{\mathbf{1}}=A \mathbf{y}_{\mathbf{1}}+\mathbf{z}_{\mathbf{1}},\left.\quad \mathbf{y}_{\mathbf{1}}\right|_{t=t_{0}}=\mathbf{0}$, where $\mathbf{y}_{\mathbf{1}}=\mathbf{y}-\mathbf{y}_{\mathbf{0}}, \quad A=$ $\left(a_{i j}\right)_{m \times n}, \quad z_{1 i}=z_{i}+\sum_{j=1}^{n} a_{i j} y_{0 j}, \quad i=1,2, \ldots, m$.

It is worth pointing out that algebraic constraints $g(\mathbf{y}, \mathbf{z})=$ 0 are represented while rows in $E$ whose elements are all 0 appear.

\section{Equivalent Transformation of LFDAE}

To discuss the theorems on the existence and uniqueness of the solution of the LFDAE introduced in Section 2, we give definition of equivalent matrix pair [14] as a necessary preparation.

Definition 1. Two pairs of matrices $\left(E_{1}, A_{1}\right)$ and $\left(E_{2}, A_{2}\right), i=$ 1,2 , are called (strongly) equivalent if there exist nonsingular matrices $P_{1} \in R^{m \times m}$ and $P_{2} \in R^{n \times n}$ such that $E_{2}=P_{1} E_{1} P_{2}$, $A_{2}=P_{1} A_{1} P_{2}$. If this is the case, one writes $\left(E_{1}, A_{1}\right) \sim\left(E_{2}, A_{2}\right)$.

Multiplying nonsingular matrix $P_{1} \in R^{m \times m}$ on the left and setting $\mathbf{y}=P_{2} \widetilde{\mathbf{y}}, P_{2} \in R^{n \times n}$ is a nonsingular matrix, LFDAE (1), where $E, A \in R^{m \times n}(m \neq n), \mathbf{y}(t) \in \mathbb{R}^{n}, \mathbf{z}(t) \in \mathbb{R}^{m}$ is transformed into

$$
\begin{aligned}
\widetilde{E}_{t_{0}} D_{t}^{(\alpha)} \widetilde{\mathbf{y}} & =\widetilde{A} \widetilde{\mathbf{y}}+\widetilde{\mathbf{z}}, \\
\left.\widetilde{\mathbf{y}}\right|_{t=t_{0}} & =\mathbf{0}, \\
& \widetilde{E}=P_{1} E P_{2}, \widetilde{A}=P_{1} A P_{2}, \widetilde{\mathbf{z}(t)}=P_{1} \mathbf{z}(t)
\end{aligned}
$$

which is again a linear fractional differential-algebraic equation with constant coefficients. System (2) could be written as $(\widetilde{E}, \widetilde{A})$ and $(E, A) \sim(\widetilde{E}, \widetilde{A})$. Because of the linearity of the operator ${ }_{t_{0}} D_{t}^{(\alpha)}$ and the nonsingularity of square matrices $P_{1}, P_{2}$, it is evident that system (2) has the same solution property as system (1). Thus, by equivalent transformation, we can consider the transformed system (2) instead of (1) with respect to solvability and related questions.

\section{Regular Matrix Pair and the Solution of LFDAE}

To simplify the problem, we now focus on the LFDAE in which $E, A$ are both square matrices; that is, $E, A \in C^{n \times n}$. Recall Definition 2 [14].

Definition 2. Let $E, A \in C^{m \times n}$, the matrix pair $(E, A)$ is called regular matrix pair if $m=n$, and the characteristic polynomial $p(\lambda)=\operatorname{det}(\lambda E-A)$ is not the zero polynomial. A matrix pair which is not regular is called singular.

Lemma 3 allows us to gain an equivalent but more simple form of the matrix pair $(E, A)$-Weierstrass canonical form [14].
Lemma 3. Let $E, A \in C^{n \times n}$ and $(E, A)$ be regular. Then, one has the Weierstrass canonical form

$$
(E, A) \sim\left(\left[\begin{array}{ll}
I & 0 \\
0 & N
\end{array}\right],\left[\begin{array}{ll}
J & 0 \\
0 & I
\end{array}\right]\right),
$$

where $J$ is a matrix in Jordan canonical form and $N$ is a nilpotent matrix also in Jordan canonical form. Moreover, it is allowed that one or the other block is not present.

Let $l$ be the index of nilpotent matrix $N \in C^{k \times k}$; that is, $N^{l}=0$ and $N^{l-1} \neq 0$ (in fact, $l$ is always less than $k$ ); we have Theorem 4 which is fundamental on the solvability of LFDAE (1).

Theorem 4. Let $l$ be the index of nilpotent matrix $N \in C^{k \times k} ; \mathbf{z}(t)$ is differentiable enough; that is, ${ }_{t_{0}} D_{t}^{(i \alpha)} \mathbf{z}(t) \quad(i=0,1,2, \ldots, l)$ is well defined, where ${ }_{t_{0}} D_{t}^{(i \alpha)}=\overbrace{{ }_{t_{0}} D_{t}^{(\alpha)}{ }_{t_{0}} D_{t}^{(\alpha)} \cdots{ }_{t_{0}} D_{t}^{(\alpha)}}^{i}$ denotes sequential fractional derivatives [11, 15-17]. Then linear fractional differentialalgebraic equation

$$
N_{t_{0}} D_{t}^{(\alpha)} \mathbf{y}(t)=\mathbf{y}(t)+\mathbf{z}(t)
$$

has unique solution $\mathbf{y}(t)=-\sum_{i=0}^{l-1} N_{t_{0}}^{i} D_{t}^{(i \alpha)} \mathbf{z}(t)$.

Proof. Firstly, we prove solutions of $(*)$ have the form of $\mathbf{y}(t)=-\sum_{i=0}^{l-1} N_{t_{0}}^{i} D_{t}^{(i \alpha)} \mathbf{z}(t)$.

Since ${ }_{t_{0}} D_{t}^{(\alpha)}$ is a linear operator maps function $\mathbf{y}(t)$ into its derivative of order $\alpha$, moving the left hand term in $(*)$ to the right, we have

$$
\left(I-N_{t_{0}} D_{t}^{(\alpha)}\right) \mathbf{y}(t)+\mathbf{z}(t)=0 .
$$

Because $N$ is a matrix consisting of constant number, it is commutable with operator $t_{0} D_{t}^{(\alpha)}$; using Neumann series and taking $l$ as the index of nilpotent matrix $N$ into account, we obtain

$$
\begin{aligned}
\mathbf{y}(t) & =-\left(I-N_{t_{0}} D_{t}^{(\alpha)}\right)^{-1} \mathbf{z}(t)=-\sum_{i=0}^{+\infty}\left(N_{t_{0}} D_{t}^{(\alpha)}\right)^{i} \mathbf{z}(t) \\
& =-\sum_{i=0}^{l-1} N^{i}{ }_{t_{0}} D_{t}^{(i \alpha)} \mathbf{z}(t)
\end{aligned}
$$

Secondly, we prove function $\mathbf{y}(t)=-\sum_{i=0}^{l-1} N^{i}{ }_{t_{0}} D_{t}^{(i \alpha)} \mathbf{z}(t)$ is the solution of $(*)$.

Substituting $\mathbf{y}(t)=-\sum_{i=0}^{l-1} N_{t_{0}}^{i} D_{t}^{(i \alpha)} \mathbf{z}(t)$ into $(*)$ yields

$$
\begin{aligned}
N_{t_{0}} D_{t}^{(\alpha)} \mathbf{y}(t)-\mathbf{y}(t)-\mathbf{z}(t) \\
=-N_{t_{0}} D_{t}^{(\alpha)}\left[\sum_{i=0}^{l-1}\left(N^{i}{ }_{t_{0}} D_{t}^{(i \alpha)}\right) \mathbf{z}(t)\right]
\end{aligned}
$$




$$
\begin{aligned}
& -\left[-\sum_{i=0}^{l-1}\left(N^{i}{ }_{t_{0}} D_{t}^{(i \alpha)}\right) \mathbf{z}(t)\right]-\mathbf{z}(t) \\
= & {\left[-\sum_{i=1}^{l-1}\left(N^{i}{ }_{t_{0}} D_{t}^{(i \alpha)}\right) \mathbf{z}(t)\right] } \\
& -\left[-\sum_{i=0}^{l-1}\left(N^{i}{ }_{t_{0}} D_{t}^{(i \alpha)}\right) \mathbf{z}(t)\right]-\mathbf{z}(t), \quad\left(N^{l}=0\right) \\
= & \mathbf{z}(t)-\mathbf{z}(t)=0 .
\end{aligned}
$$

Obviously, $\mathbf{y}(t)$ is really the solution of $(*)$.

So equation $N_{t_{0}} D_{t}^{(\alpha)} \mathbf{y}(t)=\mathbf{y}(t)+\mathbf{z}(t)$ has the unique solution $\mathbf{y}(t)=-\sum_{i=0}^{l-1} N^{i}{ }_{t_{0}} D_{t}^{(i \alpha)} \mathbf{z}(t)$.

Now let us elaborate on Theorem 5 below.

Theorem 5. Let $E, A \in R^{n \times n}, \mathbf{y}(t) ; \mathbf{z}(t) \in \mathbb{R}^{n}, 0<t<T<$ $+\infty$ and $\mathbf{y}(t), \mathbf{z}(t) \in L_{1}(0, T), \mathbf{z}(t)$ is differentiable enough; then there exists unique solution for the linear fractional differential-algebraic equations

$$
\begin{aligned}
E_{t_{0}} D_{t}^{(\alpha)} \mathbf{y} & =A \mathbf{y}+\mathbf{z}, \\
\left.\mathbf{y}\right|_{t=t_{0}} & =\mathbf{0}
\end{aligned}
$$

if the matrix pair $(E, A)$ is regular.

Proof. As claimed in Section 3, system (7) has the same solution property as system (8),

$$
\begin{aligned}
\bar{E}_{t_{0}} D_{t}^{(\alpha)} \overline{\mathbf{y}} & =\bar{A} \overline{\mathbf{y}}+\overline{\mathbf{z}}, \\
\left.\overline{\mathbf{y}}\right|_{t=t_{0}} & =\mathbf{0}, \\
\bar{E} & =P_{1} E P_{2} \in R^{n \times n}, \bar{A}=P_{1} A P_{2} \in R^{n \times n}, \overline{\mathbf{z}}=P_{1} \mathbf{z} .
\end{aligned}
$$

We now discuss the solvability of system (8). Using Lemma 3, $\bar{E}=P_{1} E P_{2}, \bar{A}=P_{1} A P_{2}$ in system (8) could be obtained in the following Weierstrass canonical form:

$$
\begin{aligned}
& \bar{E}=\left[\begin{array}{ll}
I & 0 \\
0 & N
\end{array}\right], \\
& \bar{A}=\left[\begin{array}{ll}
J & 0 \\
0 & I
\end{array}\right] .
\end{aligned}
$$

Hence system (8) appears as

$$
\begin{aligned}
{\left[\begin{array}{ll}
I & 0 \\
0 & N
\end{array}\right]{ }_{t_{0}} D_{t}^{(\alpha)} \overline{\mathbf{y}} } & =\left[\begin{array}{ll}
J & 0 \\
0 & I
\end{array}\right] \overline{\mathbf{y}}+\overline{\mathbf{z}}, \\
\left.\overline{\mathbf{y}}\right|_{t=t_{0}} & =\mathbf{0} .
\end{aligned}
$$

By setting $\overline{\mathbf{y}}=\left[\overline{\mathbf{y}_{1}}, \overline{\mathbf{y}_{2}}\right]$, system (8) is separated into two subsystems:

$$
\begin{aligned}
I_{t_{0}} D_{t}^{(\alpha)} \overline{\mathbf{y}_{1}} & =J \overline{\mathbf{y}_{1}}+\overline{\mathbf{z}_{1}} \\
\left.\overline{\mathbf{y}_{1}}\right|_{t=t_{0}} & =\mathbf{0}, \\
N_{t_{0}} D_{t}^{(\alpha)} \overline{\mathbf{y}_{2}} & =I \overline{\mathbf{y}_{2}}+\overline{\mathbf{z}_{2}} \\
\left.\overline{\mathbf{y}_{2}}\right|_{t=t_{0}} & =\mathbf{0} .
\end{aligned}
$$

Subsystem (11) is a normal fractional differential system, which has unique solution with given initial value [11]. As discussed in Theorem 4, subsystem (12) has unique solution $\overline{\mathbf{y}_{2}(t)}=-\sum_{i=0}^{l-1} N^{i}{ }_{t_{0}} D_{t}^{(i \alpha)} \overline{\mathbf{z}_{2}(t)}$.

Hence, system (8) has unique solution; accordingly system (7) has unique solution as a result. And the proof of Theorem 5 is completed.

Remark 6. Since the solution $y(t)$ in Theorem 4 is obtained without specifying the initial value of $y(t)$, initial value $\left.\overline{\mathbf{y}_{2}}\right|_{t=t_{0}}=\mathbf{0}$ is consistent when ${ }_{t_{0}} D_{t}^{(i \alpha)} \overline{\mathbf{z}_{2}\left(t_{0}\right)}=\mathbf{0}(i=$ $1,2, \ldots, l-1)$, where $l$ is the index of nilpotent matrix $N$.

Remark 7. As derivative of fractional order is the generalization of derivative of integer order, the condition "the matrix pair $(E, A)$ is regular" plays the same role as in theory of differential-algebraic equation [18].

\section{Kronecker Canonical Form and the Solution of LFDAE}

We have investigated the existence and uniqueness of the solution of LFDAE (1) in which $E, A \in R^{n \times n}$ are both square matrices. Nevertheless, the general form of LFDAE (1), where $E, A \in \mathbb{R}^{m \times n}(m \neq n)$ are both not square matrices, is frequently modeled in mechanical systems. We go on to investigate this case by tools of equivalent transformation and the Kronecker canonical form of the system.

Kronecker canonical form which is referred to as $\widetilde{E}$, $\widetilde{A}$ in Lemma 8 plays an important role in analyzing the existence and uniqueness of the solution of LFDAE. In 1974, Gantmacher pointed out Lemma $8[19,20]$.

Lemma 8. Let $A \in R^{m \times n}$; then there exist nonsingular matrices $P \in R^{m \times m}$ and $Q \in R^{n \times n}$, such that $(E, A) \sim(\widetilde{E}, \widetilde{A})$ $(P E Q=\widetilde{E}, P A Q=\widetilde{A})$, where $\widetilde{E}, \widetilde{A}$ take the next form:

$$
\begin{aligned}
\widetilde{E} & =P E Q \\
& =\operatorname{diag}\left(\mathbf{0}_{n_{0} \times n_{0}}, L_{1}, L_{2}, \ldots, L_{p}, \widetilde{L_{1}}, \widetilde{L_{2}}, \ldots, \widetilde{L_{q}}, I, N\right), \\
\widetilde{A} & =P A Q \\
& =\operatorname{diag}\left(\mathbf{0}_{n_{0} \times n_{0}}, J_{1}, J_{2}, \ldots, J_{p}, \widetilde{J_{1}}, \widetilde{J_{2}}, \ldots, \widetilde{J_{q}}, A_{1}, I\right),
\end{aligned}
$$




$$
\begin{aligned}
& L_{i}=\left[\begin{array}{ccccc}
1 & 0 & & & \\
& 1 & 0 & & \\
& & \ddots & \ddots & \\
& & & 1 & 0
\end{array}\right], \\
& J_{i}=\left[\begin{array}{ccccc}
0 & 1 & & & \\
& 0 & 1 & & \\
& & \ddots & \ddots & \\
& & & 0 & 1
\end{array}\right] \in \mathbb{R}^{n_{i} \times\left(n_{i}+1\right)} \\
& \widetilde{\mathbf{y}(t)}=\left[y_{0}{ }^{T} ; y_{L 1}{ }^{T}, y_{L 2}{ }^{T}, \ldots, y_{L p}{ }^{T} ; y_{\widetilde{L} 1}{ }^{T}, y_{\widetilde{L} 2}{ }^{T}, \ldots, y_{\widetilde{L} q}{ }^{T} ;\right. \\
& \left.y_{I}^{T} ; y_{N 1}^{T}, y_{N 2}{ }^{T}, \ldots, y_{N l}{ }^{T}\right]^{\top} ; \\
& \widetilde{\mathbf{z}(t)}=\left[z_{0}{ }^{T} ; z_{L 1}{ }^{T}, z_{L 2}{ }^{T}, \ldots, z_{L p}{ }^{T} ; z_{\widetilde{L} 1}{ }^{T}, z_{\widetilde{L} 2}{ }^{T}, \ldots, z_{\widetilde{L} q}{ }^{T} ; z_{I}{ }^{T} ;\right. \\
& \left.z_{N 1}^{T}, z_{N 2}{ }^{T}, \ldots, z_{N l}{ }^{T}\right]^{\top} \text {. }
\end{aligned}
$$$$
\widetilde{L_{j}}=\left[\begin{array}{cccc}
1 & & & \\
0 & 1 & & \\
& \ddots & \ddots & \\
& & & 1 \\
& & & 0
\end{array}\right]
$$$$
\widetilde{J_{j}}=\left[\begin{array}{cccc}
0 & & & \\
1 & 0 & & \\
& \ddots & \ddots & \\
& & & 0 \\
& & & 1
\end{array}\right] \in \mathbb{R}^{\left(\widetilde{n_{j}}+1\right) \times \widetilde{n_{j}}}
$$$$
i=1,2, \ldots, p, j=1,2, \ldots, q \text {, }
$$$$
N=\operatorname{diag}\left(N_{1}, N_{2}, \ldots, N_{l}\right) \in \mathbb{R}^{h \times h},
$$$$
N_{s}=\left[\begin{array}{ccccc}
0 & 1 & & & \\
& 0 & 1 & & \\
& & \ddots & \ddots & \\
& & & 0 & 1 \\
& & & & 0
\end{array}\right] \in \mathbb{R}^{k_{s} \times k_{s}}
$$$$
A_{1}, I \in \mathbb{R}^{g \times g} .
$$

\section{Dimension of each matrix satisfies}

$$
\begin{array}{r}
n_{0}+\sum_{i} n_{i}+\sum_{j}\left(\widetilde{n_{j}}+1\right)+\sum_{s} k_{s}+g=m ; \\
n_{0}+\sum_{i}\left(n_{i}+1\right)+\sum_{j} \widetilde{n_{j}}+\sum_{s} k_{s}+g=n ; \\
\sum_{s} k_{s}=h .
\end{array}
$$

According to Lemma 8, setting $P_{1}=P, P_{2}=Q, \widetilde{E}, \widetilde{A}$ in system (2) transformed from system (1) have the Kronecker canonical form through equivalent transformation: $\mathbf{y}=Q \widetilde{\mathbf{y}}$, $\widetilde{\mathbf{z}(t)}=P \mathbf{z}(t)$.
Hence, LFDAE (2) could be transformed into the following equivalent equations:

$$
\begin{aligned}
\mathbf{0}_{n_{0} \times n_{0} t_{0}} D_{t}^{(\alpha)} y_{0}(t) & =z_{0}(t), \\
L_{i_{t_{0}}} D_{t}^{(\alpha)} y_{L i}(t) & =J_{i} y_{L i}(t)+z_{L i}(t), \\
& i=1,2, \ldots, p ; \\
\widetilde{L}_{j t_{0}} D_{t}^{(\alpha)} y_{\widetilde{L}_{j}}(t) & =\widetilde{J}_{j} y_{\widetilde{L}_{j}}(t)+z_{\widetilde{L}_{j}}(t), \\
& j=1,2, \ldots, q ; \\
N_{s} D_{t}^{(\alpha)} y_{N s}(t) & =y_{N s}(t)+z_{N s}(t), \\
& s=1,2, \ldots, l,
\end{aligned}
$$

and the equation

$$
D_{t}^{(\alpha)} y_{I}(t)=A_{1} y_{I}(t)+z_{I}(t)
$$

Now, let us discuss (16)-(20) to investigate the existence and uniqueness of the solution of $\operatorname{LFDAE}(2)$ in which $\widetilde{E}, \widetilde{A}$ take the Kronecker canonical form.

With regard to (16), it is easy to see that the equation is solvable if and only if all components of $z_{0}(t)$ are zeros; that is, $z_{0}(t)=\mathbf{0}$. In this case, (16) either has no solution while $z_{0}(t) \neq$ $\mathbf{0}$ or has solutions of infinite number (as long as $z_{0}(t)=\mathbf{0}$, any function that is differentiable of order $\alpha$ could be considered as the solution of (16)). So there exists no unique solution for (16).

With regard to (17), considering the particular form of $L_{i}$, $J_{i}$, (17) appears as

$$
\begin{aligned}
& {\left[\begin{array}{lllll}
1 & 0 & & & \\
& 1 & 0 & & \\
& & \ddots & \ddots & \\
& & & 1 & 0
\end{array}\right]{ }_{t_{0}} D_{t}^{(\alpha)} y_{L i}(t)} \\
& =\left[\begin{array}{lllll}
0 & 1 & & & \\
& 0 & 1 & \\
& & \ddots & \ddots & \\
& & & 0 & 1
\end{array}\right] y_{L i}(t)+z_{L i}(t),
\end{aligned}
$$


Denoting components of $y_{L i}(t)$ as $\left(y_{L i}{ }^{(1)}, y_{L i}{ }^{(2)}, \ldots\right.$, $\left.y_{L i}{ }^{\left(n_{i}+1\right)}\right)^{T}$ and $z_{L i}(t)$ as $\left(z_{L i}{ }^{(1)}, z_{L i}{ }^{(2)}, \ldots, z_{L i}{ }^{\left(n_{i}\right)}\right)^{T}$, the above equation is precisely equal to

$$
\begin{gathered}
{ }_{t_{0}} D_{t}^{(\alpha)} y_{L i}{ }^{(1)}(t)=y_{L i}{ }^{(2)}(t)+z_{L i}{ }^{(1)}(t), \\
{ }_{t_{0}} D_{t}^{(\alpha)} y_{L i}{ }^{(2)}(t)=y_{L i}{ }^{(3)}(t)+z_{L i}{ }^{(2)}(t), \\
\vdots \\
{ }_{t_{0}} D_{t}^{(\alpha)} y_{L i}{ }^{\left(n_{i}\right)}(t)=y_{L i}{ }^{\left({ }^{(}+1\right)}(t)+z_{L i}{ }^{\left(n_{i}\right)}(t), \\
i=1,2, \ldots, p .
\end{gathered}
$$

We further have

$$
\begin{gathered}
y_{L i}{ }^{(2)}(t)={ }_{t_{0}} D_{t}^{(\alpha)} y_{L i}{ }^{(1)}(t)-z_{L i}{ }^{(1)}(t), \\
y_{L i}{ }^{(3)}(t)={ }_{t_{0}} D_{t}^{(\alpha)} y_{L i}{ }^{(2)}(t)-z_{L i}{ }^{(2)}(t), \\
\vdots \\
y_{L i}{ }^{\left(n_{i}+1\right)}(t)={ }_{t_{0}} D_{t}^{(\alpha)} y_{L i}{ }^{\left(n_{i}\right)}(t)-z_{L i}{ }^{\left(n_{i}\right)}(t), \\
i=1,2, \ldots, p .
\end{gathered}
$$

Since $y_{L i}{ }^{(1)}(t)$ in (23) is free of all restrictions, any function differentiable of order $\alpha$ could be considered as $y_{L i}{ }^{(1)}(t)$; thus (17) has solutions of infinite number.

With regard to (18), considering the particular form of matrices $\widetilde{L_{j}}, \widetilde{J_{j}}$, (18) appears as

$$
\begin{aligned}
& {\left[\begin{array}{cccc}
1 & & & \\
0 & 1 & & \\
& \ddots & \ddots & \\
& & & 1 \\
& & & 0
\end{array}\right] t_{0} D_{t}^{(\alpha)} y_{\widetilde{L j}}(t)} \\
& =\left[\begin{array}{cccc}
0 & & & \\
1 & 0 & & \\
& \ddots & \ddots & \\
& & & 0 \\
& & & 1
\end{array}\right] y_{\widetilde{L j}}(t)+z_{\widetilde{L j}}(t), \\
& j=1,2, \ldots, q, \widetilde{L_{j}}, \widetilde{J_{j}} \in \mathbb{R}^{\left(\widetilde{n_{j}}+1\right) \times \widetilde{n_{j}}} .
\end{aligned}
$$

Denoting components of $y_{\widetilde{L j}}(t)$ as $\left(y_{\widetilde{L j}}{ }^{(1)}, y_{\widetilde{L j}}{ }^{(2)}, \ldots\right.$, $\left.y_{\widetilde{L j}}{ }^{\left(\widetilde{n_{j}}\right)}\right)^{T}$ and $z_{\widetilde{L j}}(t)$ as $\left(z_{\widetilde{L j}}{ }^{(1)}, z_{\widetilde{L j}}{ }^{(2)}, \ldots, z_{\widetilde{L j}}{ }^{\left(\widetilde{n_{j}}+1\right)}\right)^{T}$, the above equation is precisely equal to

$$
\begin{gathered}
t_{0} D_{t}^{(\alpha)} y_{\widetilde{L j}}{ }^{(1)}(t)=z_{\widetilde{L j}}{ }^{(1)}(t), \\
t_{0} D_{t}^{(\alpha)} y_{\widetilde{L j}}{ }^{(2)}(t)=y_{\widetilde{L j}}{ }^{(1)}(t)+z_{\widetilde{L j}}{ }^{(2)}(t),
\end{gathered}
$$

$$
\begin{aligned}
{ }_{t_{0}} D_{t}^{(\alpha)} y_{\widetilde{L j}}{ }^{\left(\widetilde{n_{j}}\right)}(t)=y_{\widetilde{L j}}{ }^{\left(\widetilde{n_{j}}-1\right)}(t)+z_{\widetilde{L j}}^{\left(\widetilde{n_{j}}\right)}(t), \\
\quad j=1,2, \ldots, q,
\end{aligned}
$$

and an additional formula

$$
y_{\widetilde{L j}}^{\left(\widetilde{n_{j}}\right)}(t)+z_{\widetilde{L j}}^{\left(\widetilde{n_{j}}+1\right)}(t)=0
$$

from which we get

$$
y_{\widetilde{L j}}^{\left(\widetilde{n_{j}}\right)}(t)=-z_{\widetilde{L j}}^{\left(\widetilde{n_{j}}+1\right)}(t) .
$$

Thus 5 could be rewritten in a recursive form of reverse order:

$$
\begin{gathered}
t_{0} D_{t}^{(\alpha)} y_{\widetilde{L j}}{ }^{(1)}(t)=z_{\widetilde{L j}}{ }^{(1)}(t), \\
y_{\widetilde{L j}}{ }^{(1)}(t)={ }_{t_{0}} D_{t}^{(\alpha)} y_{\widetilde{L j}}{ }^{(2)}(t)-z_{\widetilde{L j}}{ }^{(2)}(t), \\
\vdots \\
y_{\widetilde{L j}}{ }^{\left(\widetilde{n_{j}}-1\right)}(t)={ }_{t_{0}} D_{t}^{(\alpha)} y_{\widetilde{L j}}{ }^{\left(\widetilde{n_{j}}\right)}(t)-z_{\widetilde{L j}}{ }^{\left(\widetilde{n_{j}}\right)}(t), \\
\quad j=1,2, \ldots, q .
\end{gathered}
$$

Obviously, to any given differential enough $\mathbf{z}_{\widetilde{L j}}(t), 5$ has unique solution $\mathbf{y}_{\widetilde{L j}}(t)$. But the obtained $y_{\widetilde{L j}}{ }^{(1)}(t)$ hardly satisfy the equation ${ }_{t_{0}} D_{t}^{(\alpha)} y_{\widetilde{L j}}{ }^{(1)}(t)=z_{\widetilde{L j}}{ }^{(1)}(t)$ in 5 except very special situation. Therefore, (18) has no feasible solution for any given $\mathbf{z}_{\widetilde{L j}}(t)$.

With regard to (19), considering the particular form of matrices $N_{s}$, (19) appears as

$$
\begin{aligned}
& {\left[\begin{array}{ccccc}
0 & 1 & & & \\
& 0 & 1 & & \\
& & \ddots & \ddots & \\
& & & 0 & 1 \\
& & & & 0
\end{array}\right] D_{t}^{(\alpha)} y_{N s}(t)=y_{N s}(t)+z_{N s}(t)} \\
& s=1,2, \ldots, l ; N_{s} \in \mathbb{R}^{k_{s} \times k_{s}} .
\end{aligned}
$$

Denoting components of $y_{N s}(t)$ as $\left(y_{N s}{ }^{(1)}, y_{N s}{ }^{(2)}, \ldots\right.$, $\left.y_{N s}{ }^{\left(k_{s}\right)}\right)^{T}$ and $z_{N s}(t)$ as $\left(z_{N s}{ }^{(1)}, z_{N s}{ }^{(2)}, \ldots, z_{N s}{ }^{\left(k_{s}\right)}\right)^{T}$, the above equation is equal to

$$
\begin{gathered}
{ }_{t_{0}} D_{t}^{(\alpha)} y_{N s}{ }^{(2)}(t)=y_{N s}{ }^{(1)}(t)+z_{N s}{ }^{(1)}(t), \\
{ }_{t_{0}} D_{t}^{(\alpha)} y_{N s}{ }^{(3)}(t)=y_{N s}{ }^{(2)}(t)+z_{N s}{ }^{(2)}(t), \\
\vdots \\
{ }_{t_{0}} D_{t}^{(\alpha)} y_{N s}{ }^{\left(k_{s}\right)}(t)=y_{N s}{ }^{\left(k_{s}-1\right)}(t)+z_{N s}{ }^{\left(k_{s}-1\right)}(t), \\
y_{N s}{ }^{\left(k_{s}\right)}(t)+z_{N s}{ }^{\left(k_{s}\right)}(t)=0, \\
s=1,2, \ldots, l .
\end{gathered}
$$


We further obtain the equivalence of (28) as below:

$$
\begin{gathered}
y_{N s}{ }^{(1)}(t)={ }_{t_{0}} D_{t}^{(\alpha)} y_{N s}{ }^{(2)}(t)-z_{N s}{ }^{(1)}(t), \\
y_{N s}{ }^{(2)}(t)={ }_{t_{0}} D_{t}^{(\alpha)} y_{N s}{ }^{(3)}(t)-z_{N s}{ }^{(2)}(t), \\
\vdots \\
y_{N s}{ }^{\left(k_{s}-1\right)}(t)={ }_{t_{0}} D_{t}^{(\alpha)} y_{N s}{ }^{\left(k_{s}\right)}(t)-z_{N s}{ }^{\left(k_{s}-1\right)}(t), \\
y_{N s}{ }^{\left(k_{s}\right)}(t)=-z_{N s}{ }^{\left(k_{s}\right)}(t), \quad s=1,2, \ldots, l .
\end{gathered}
$$

Obviously, to any given $\mathbf{z}_{N s}(t)$, solution $\mathbf{y}_{N s}(t)$ could be derived from (29) if $\mathbf{z}(t)$ is differentiable enough; that is, ${ }_{t_{0}} D_{t}^{(i \alpha)} \mathbf{z}$ is well defined. Thus, (19) always has unique solution towards any given suitable vector $\mathbf{z}_{N s}(t)$.

With regard to (20), where $E=I \in \mathbb{R}^{g \times g}, A_{1} \in \mathbb{R}^{g \times g}$ are square matrices, because of Theorem 5, (20) has unique solution if the matrix pair $\left(I, A_{1}\right)$ is regular.

Bringing the analysis above together, LFDAE (2) has unique solution if (16) to (18) do not appear in their Kronecker canonical form, while matrix pair $\left(I, A_{1}\right)$ is regular. The above-mentioned summarization brings us to Theorem 9.

Theorem 9. Let $E, A \in \mathbb{R}^{m \times n}$. The linear fractional differential-algebraic equations

$$
\begin{aligned}
E_{t_{0}} D_{t}^{(\alpha)} \mathbf{y} & =A \mathbf{y}+b \mathbf{z} \\
\left.\mathbf{y}\right|_{t=t_{0}} & =\mathbf{0}
\end{aligned}
$$

in which $\mathbf{y}(t) \in \mathbb{R}^{n}, \mathbf{z}(t) \in \mathbb{R}^{m}, 0<t<T<+\infty$ satisfy the following:

(1) $\mathbf{y}(t), \mathbf{z}(t) \in L_{1}(0, T)$;

(2) ${ }_{t_{0}} D_{t}^{(i \alpha)} \mathbf{z}(i=0,1,2, \ldots, n)$ is well defined, and ${ }_{t_{0}} D_{t}^{(i \alpha)} \mathbf{z}(0)=\mathbf{0},(i=0,1,2, \ldots, n)$.

Then, the system has unique solution if there exist nonsingular matrices $P \in R^{m \times m}$ and $Q \in R^{n \times n}$, such that $(E, A) \sim$ $(\widetilde{E}, \widetilde{A})$, where $\widetilde{E}=\operatorname{diag}(I, N), \widetilde{A}=\operatorname{diag}\left(A_{1}, I\right)$, and matrix pair $\left(I, A_{1}\right)$ is regular.

Remark 10. Theorem 9 is also suitable when other fractional derivatives are involved. Since ${ }_{t_{0}} D_{t}^{(\alpha)}$ in the sequential fractional derivatives in Theorem 4 can mean the RiemannLiouville, the Grünwald-Letnikov, the Caputo, or even any other definition of fractional derivatives [11], Theorem 5 is applicable to these fractional derivatives. Meanwhile, analysis in Section 5 is still valid for other definitions of fractional derivatives, so it is the same as Theorem 9 as a result.

\section{Examples}

Now let us take some examples on the theorems represented before.
Example 1. LFDAE in which $E=N$ is a nilpotent matrix and $A=I$ is as below (example of Theorem 4 ):

$$
\begin{aligned}
& \left(\begin{array}{lll}
0 & 1 & 2 \\
0 & 0 & 3 \\
0 & 0 & 0
\end{array}\right)\left(\begin{array}{cc}
{ }_{0} D_{t}^{(0.5)} & y_{1}(t) \\
{ }_{0} D_{t}^{(0.5)} & y_{2}(t) \\
{ }_{0} D_{t}^{(0.5)} & y_{3}(t)
\end{array}\right) \\
& =\left(\begin{array}{lll}
1 & 0 & 0 \\
0 & 1 & 0 \\
0 & 0 & 1
\end{array}\right)\left(\begin{array}{l}
y_{1}(t) \\
y_{2}(t) \\
y_{3}(t)
\end{array}\right)+\left(\begin{array}{c}
t^{2.5} \\
t^{2} \\
t^{1.5}
\end{array}\right) \text {, } \\
& \mathbf{y}(0)=\mathbf{0} \text {. }
\end{aligned}
$$

In the example, $E=\left(\begin{array}{lll}0 & 1 & 2 \\ 0 & 0 & 3 \\ 0 & 0 & 0\end{array}\right)=N$ is a nilpotent matrix with index $l=3, A=\left(\begin{array}{lll}1 & 0 & 0 \\ 0 & 1 & 0 \\ 0 & 0 & 1\end{array}\right)=I$. So according to Theorem 4 , the unique solution of Example 1 is

$$
\begin{aligned}
\mathbf{y}(t) & =-\sum_{i=0}^{l-1} N_{0}^{i} D_{t}^{(i \times 0.5)} \mathbf{z}(t)=-\left(\mathbf{z}(t)+N_{0} D_{t}^{(0.5)} \mathbf{z}(t)\right. \\
+ & \left.N^{2}{ }_{0} D_{t}^{(2 \times 0.5)} \mathbf{z}(t)\right)=-\left(\left(\begin{array}{c}
t^{2.5} \\
t^{2} \\
t^{1.5}
\end{array}\right)\right.
\end{aligned}
$$$$
+\left(\begin{array}{lll}
0 & 1 & 2 \\
0 & 0 & 3 \\
0 & 0 & 0
\end{array}\right){ }_{0} D_{t}^{(0.5)}\left(\begin{array}{c}
t^{2.5} \\
t^{2} \\
t^{1.5}
\end{array}\right)
$$$$
\left.+\left(\begin{array}{lll}
0 & 1 & 2 \\
0 & 0 & 3 \\
0 & 0 & 0
\end{array}\right)^{2} D_{t}^{(2 \times 0.5)}\left(\begin{array}{c}
t^{2.5} \\
t^{2} \\
t^{1.5}
\end{array}\right)\right)=-\left(\left(\begin{array}{c}
t^{2.5} \\
t^{2} \\
t^{1.5}
\end{array}\right)\right.
$$$$
+\left(\begin{array}{lll}
0 & 1 & 2 \\
0 & 0 & 3 \\
0 & 0 & 0
\end{array}\right)\left(\begin{array}{c}
\frac{\Gamma(3.5)}{\Gamma(3)} t^{2} \\
\frac{\Gamma(3)}{\Gamma(2.5)} t^{1.5} \\
\frac{\Gamma(2.5)}{\Gamma(2)} t
\end{array}\right)
$$

$$
\left.\begin{array}{l}
+\left(\begin{array}{lll}
0 & 0 & 3 \\
0 & 0 & 0 \\
0 & 0 & 0
\end{array}\right)\left(\begin{array}{c}
\frac{\Gamma(3.5)}{\Gamma(2.5)} t^{1.5} \\
\frac{\Gamma(3)}{\Gamma(2)} t \\
\frac{\Gamma(2.5)}{\Gamma(1.5)} t^{0.5}
\end{array}\right)
\end{array}\right) .
$$




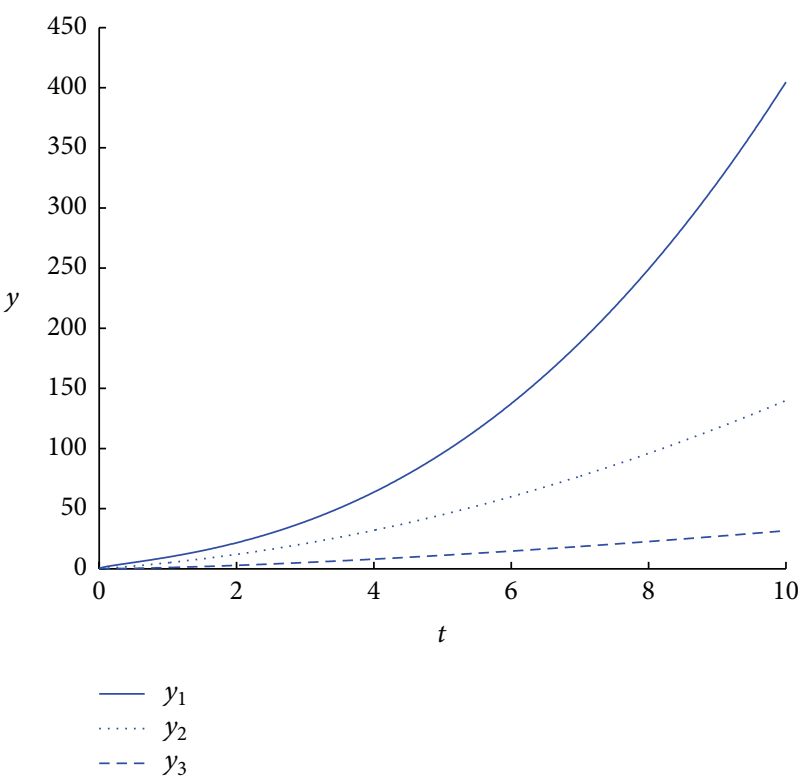

Figure 1: Solution of Example 1.

Figure 1 shows the solution of Example 1. The correctness and the initial value consistency of the solution could be verified by computation.

Example 2. LFDAE with Kronecker canonical form is as below (example of Theorem 5):

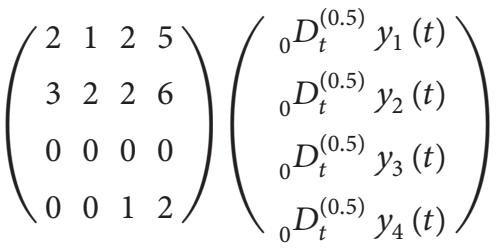

$$
\begin{aligned}
& =\left(\begin{array}{llll}
4 & 3 & 5 & 11 \\
5 & 4 & 5 & 13 \\
0 & 0 & 1 & 2 \\
0 & 0 & 3 & 5
\end{array}\right)\left(\begin{array}{l}
y_{1}(t) \\
y_{2}(t) \\
y_{3}(t) \\
y_{4}(t)
\end{array}\right)+\left(\begin{array}{c}
2 t \\
2 t \\
t \\
2 t
\end{array}\right)
\end{aligned}
$$

with the initial value $\mathbf{y}(0)=\mathbf{0}$.

$$
\text { In this example, } E=\left(\begin{array}{cccc}
2 & 1 & 2 & 5 \\
3 & 2 & 2 & 6 \\
0 & 0 & 0 & 0 \\
0 & 0 & 1 & 2
\end{array}\right), A=\left(\begin{array}{cccc}
4 & 3 & 5 & 11 \\
5 & 4 & 5 & 13 \\
0 & 0 & 1 & 2 \\
0 & 0 & 3 & 5
\end{array}\right) \text {; there }
$$
exist two nonsingular matrices $P$ and $Q$ :

$$
\begin{aligned}
P & =\left(\begin{array}{cccc}
1 & 0 & 3 & -2 \\
-1 & 1 & -2 & 1 \\
0 & 0 & -2 & 1 \\
0 & 0 & 1 & 0
\end{array}\right), \\
Q & =\left(\begin{array}{cccc}
1 & -1 & 0 & 1 \\
-1 & 2 & 1 & -3 \\
0 & 0 & 2 & -1 \\
0 & 0 & -1 & 1
\end{array}\right),
\end{aligned}
$$

satisfying

$$
\begin{aligned}
& \widetilde{E}=P E Q=\left(\begin{array}{llll}
1 & 0 & 0 & 0 \\
0 & 1 & 0 & 0 \\
0 & 0 & 0 & 1 \\
0 & 0 & 0 & 0
\end{array}\right), \\
& \widetilde{A}=P A Q=\left(\begin{array}{llll}
1 & 2 & 0 & 0 \\
0 & 1 & 0 & 0 \\
0 & 0 & 1 & 0 \\
0 & 0 & 0 & 1
\end{array}\right), \\
& \widetilde{Z}=P Z=\left(\begin{array}{l}
t \\
0 \\
0 \\
t
\end{array}\right) .
\end{aligned}
$$

Obviously, $\widetilde{E}=\operatorname{diag}(I, N), \widetilde{A}=\operatorname{diag}\left(A_{1}, I\right)$; we further get $\left|\lambda I-A_{1}\right|=\left|\begin{array}{cc}\lambda-1 & -2 \\ 0 & \lambda-1\end{array}\right|=(\lambda-1)^{2}$, which is not a zero polynomial; hence the matrix pair $\left(I, A_{1}\right)$ is regular.

From Theorem 9, the fractional system has unique solution. Now let us find the solution.

By setting $\mathbf{y}(t)=Q \overline{\mathbf{y}(t)}$, that is, $\widetilde{\mathbf{y}(t)}=Q^{-1} \mathbf{y}(t)$, multiplying $P$ on the left to (a), we obtain the equivalent transformation of (a) in the next form:

$$
\begin{gathered}
\left(\begin{array}{llll}
1 & 0 & 0 & 0 \\
0 & 1 & 0 & 0 \\
0 & 0 & 0 & 1 \\
0 & 0 & 0 & 0
\end{array}\right)\left(\begin{array}{l}
{ }_{0} D_{t}^{(0.5)} \widetilde{y_{1}(t)} \\
{ }_{0} D_{t}^{(0.5)} \widetilde{y_{2}(t)} \\
{ }_{0} D_{t}^{(0.5)} \overline{y_{3}(t)} \\
{ }_{0} D_{t}^{(0.5)} \overline{y_{4}(t)}
\end{array}\right) \\
=\left(\begin{array}{llll}
1 & 2 & 0 & 0 \\
0 & 1 & 0 & 0 \\
0 & 0 & 1 & 0 \\
0 & 0 & 0 & 1
\end{array}\right)\left(\begin{array}{l}
\widetilde{y_{1}(t)} \\
\widetilde{y_{2}(t)} \\
\widetilde{y_{3}(t)}
\end{array}\right)+\left(\begin{array}{l}
t \\
0 \\
0 \\
t
\end{array}\right),
\end{gathered}
$$

$$
\widetilde{\mathbf{y}}(0)=\mathbf{0}
$$

It is easy to obtain the unique solution for (b) by Laplace transform [11]:

$$
\begin{aligned}
& \widetilde{y_{1}(t)}=t^{3 / 2} E_{1 / 2,5 / 2}(\sqrt{t})=\sum_{k=0}^{+\infty} \frac{t^{(k+3) / 2}}{\Gamma((k+5) / 2)}, \\
& \widetilde{y_{2}(t)}=0, \\
& \widetilde{y_{3}(t)}=-\frac{2}{\sqrt{\pi}} \sqrt{t}, \\
& \widetilde{y_{4}(t)}=-t .
\end{aligned}
$$



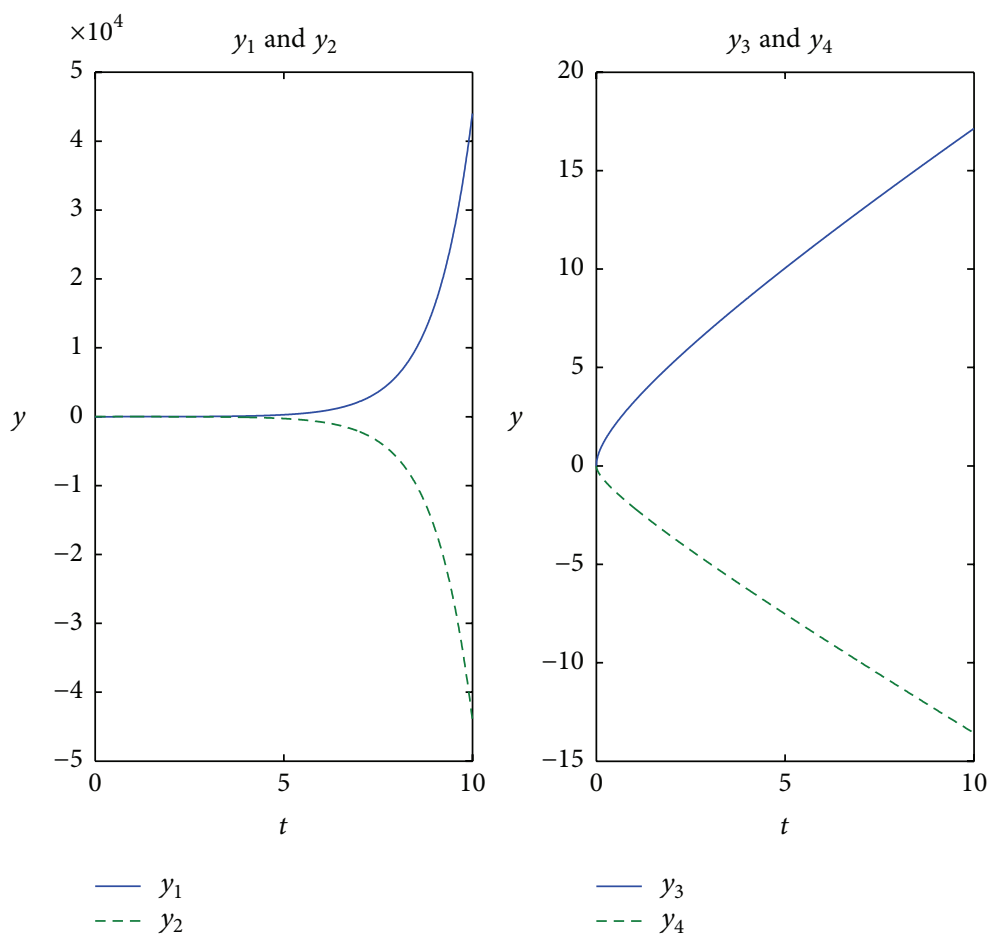

FIGURE 2: Solution of Example 2.

Accordingly, the solution for LFDAE (a) is

$$
\begin{aligned}
& y_{1}(t)=\sum_{k=0}^{+\infty} \frac{t^{(k+3) / 2}}{\Gamma((k+5) / 2)}-t, \\
& y_{2}(t)=-\sum_{k=0}^{+\infty} \frac{t^{(k+3) / 2}}{\Gamma((k+5) / 2)}-\frac{2}{\sqrt{\pi}} \sqrt{t}+3 t, \\
& y_{3}(t)=-\frac{4}{\sqrt{\pi}} \sqrt{t}+t \\
& y_{4}(t)=\frac{2}{\sqrt{\pi}} \sqrt{t}-t .
\end{aligned}
$$

And the solution satisfies $\mathbf{y}(0)=\mathbf{0}$. The solution of Example 2 is shown in Figure 2.

\section{Competing Interests}

The authors declare that they have no competing interests.

\section{Acknowledgments}

This project is supported by the National Natural Science Foundation of China (no. 11272159).

\section{References}

[1] K. Adolfsson, "Nonlinear fractional order viscoelasticity at large strains," Nonlinear Dynamics, vol. 38, no. 1-4, pp. 233-246, 2004.
[2] F. Padula, S. Alcántara, R. Vilanova, and A. Visioli, " $H_{\infty}$ control of fractional linear systems," Automatica, vol. 49, no. 7, pp. 22762280, 2013.

[3] H.-S. Wang, W.-L. Jhu, C.-F. Yung, and P.-F. Wang, "Numerical solutions of differential-algebraic equations and its applications in solving TPPC problems," Journal of Marine Science and Technology, vol. 19, no. 1, pp. 76-88, 2011.

[4] P. Fereydoun, M. Mansoureh, K. Arash et al., "The application of fractional differential equation to mortgage problem," Journal of the American Chemical Society, vol. 2, no. 4, pp. 227-231, 2013.

[5] M. Zingales, "Fractional-order theory of heat transport in rigid bodies," Communications in Nonlinear Science \& Numerical Simulation, vol. 19, no. 11, pp. 3938-3953, 2014.

[6] Z. Hu, W. Liu, and J. Liu, "Two-point boundary value problems for fractional differential equations at resonance," Bulletin of the Malaysian Mathematical Society, vol. 3, no. 3, pp. 747-755, 2014.

[7] B. Ibis and M. Bayram, "Numerical comparison of methods for solving fractional differential-algebraic equations (FDAEs)," Computers \& Mathematics with Applications, vol. 62, no. 8, pp. 3270-3278, 2011.

[8] X.-L. Ding and Y.-L. Jiang, "Waveform relaxation method for fractional differential-algebraic equations," Fractional Calculus and Applied Analysis, vol. 17, no. 3, pp. 585-604, 2014.

[9] B. Ibis, M. Bayram, and A. G. Agargun, "Applications of fractional differential transform method to fractional differentialalgebraic equations," European Journal of Pure \& Applied Mathematics, vol. 4, no. 2, pp. 129-141, 2011.

[10] Z. Y. Feng and N. Chen, "On the solution of fractional differential-algebraic systems with the adomian decomposition method," Applied Mathematics \& Mechanics, no. 36, pp. 12111218, 2015.

[11] I. Podlubny, Fractional Differential Equations, vol. 198, Academic Press, San Diego, Calif, USA, 1999. 
[12] T. Abdeljawad, "On Riemann and Caputo fractional differences," Computers \& Mathematics with Applications, vol. 62, no. 3, pp. 1602-1611, 2011.

[13] R. Almeida and D. F. M. Torres, "Necessary and sufficient conditions for the fractional calculus of variations with Caputo derivatives," Communications in Nonlinear Science \& Numerical Simulation, vol. 16, no. 3, pp. 1490-1500, 2011.

[14] P. Kunkel and V. Mehrmann, Differential-Algebraic EquationsAnalysis and Numerical Solution, European Mathematical Society, Prague, Czech Republic, 2006.

[15] A. Chikrii and I. Matychyn, "Riemann-Liouville, Caputo, and sequential fractional derivatives in differential games," Annals of the International Society of Dynamic Games, vol. 11, pp. 61-81, 2010.

[16] K. M. Furati, "A Cauchy-type problem with a sequential fractional derivative in the space of continuous functions," Boundary Value Problems, vol. 2012, no. 2, article 58, 14 pages, 2012.

[17] G. B. Loghmani and S. Javanmardi, "Numerical methods for sequential fractional differential equations for Caputo operator," Bulletin of the Malaysian Mathematical Sciences Society, vol. 35, no. 2, pp. 315-323, 2012.

[18] K. E. Brenan, S. L. Campbell, and L. R. Petzold, Numerical Solution of Initial-Value Problems in Differential-Algebraic Equations, Society for Industrial and Applied Mathematics, Philadelphia, Pa, USA, 1996.

[19] G. R. Duan, Analysis and Design of Descriptor Linear Systems, Springer, New York, NY, USA, 2010.

[20] F. R. Gantmacher, The Theory of Matrices, vol. II, Chelsea, New York, NY, USA, 1974. 


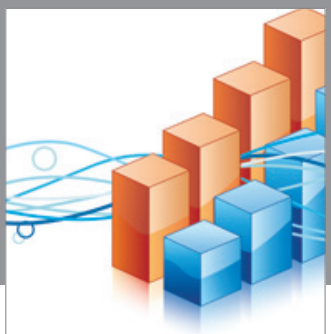

Advances in

Operations Research

vatem alat4

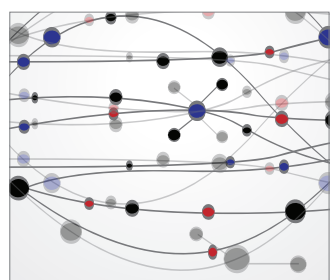

\section{The Scientific} World Journal
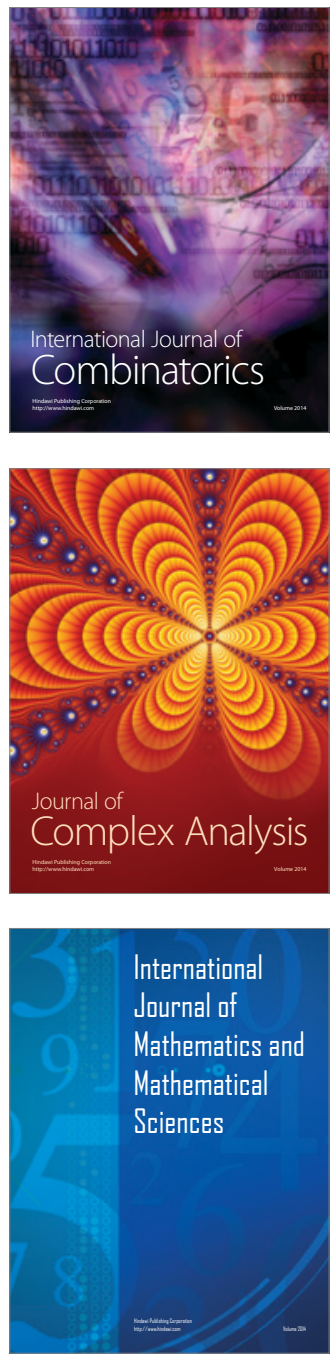
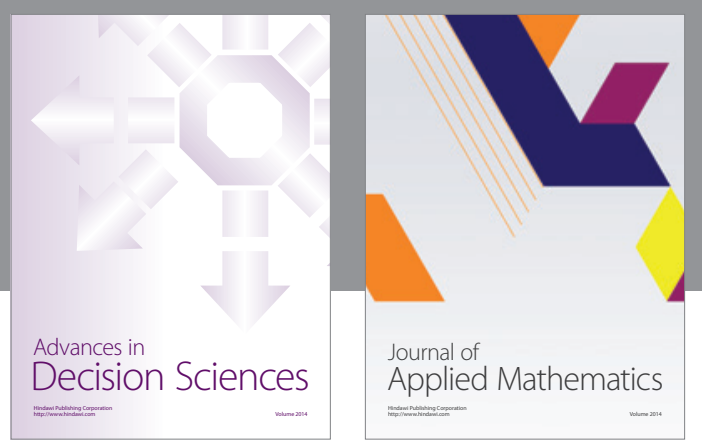

Algebra

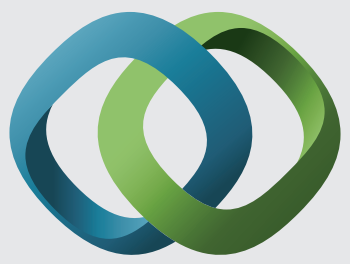

\section{Hindawi}

Submit your manuscripts at

http://www.hindawi.com
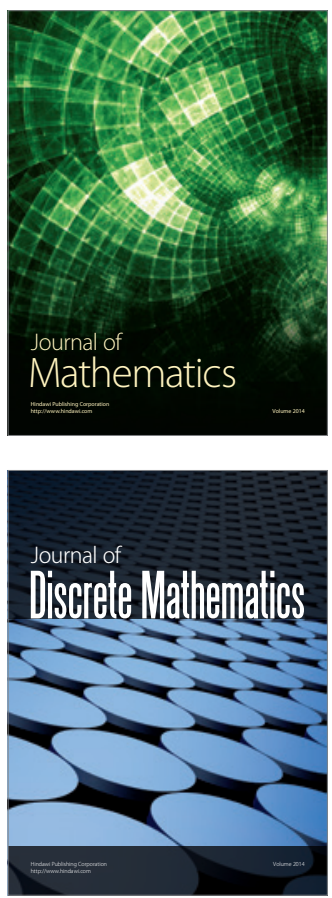

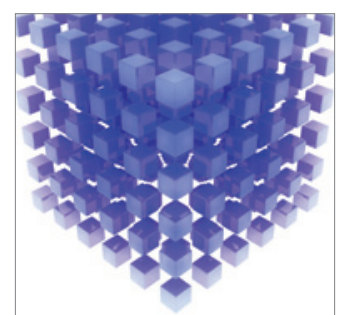

Mathematical Problems in Engineering
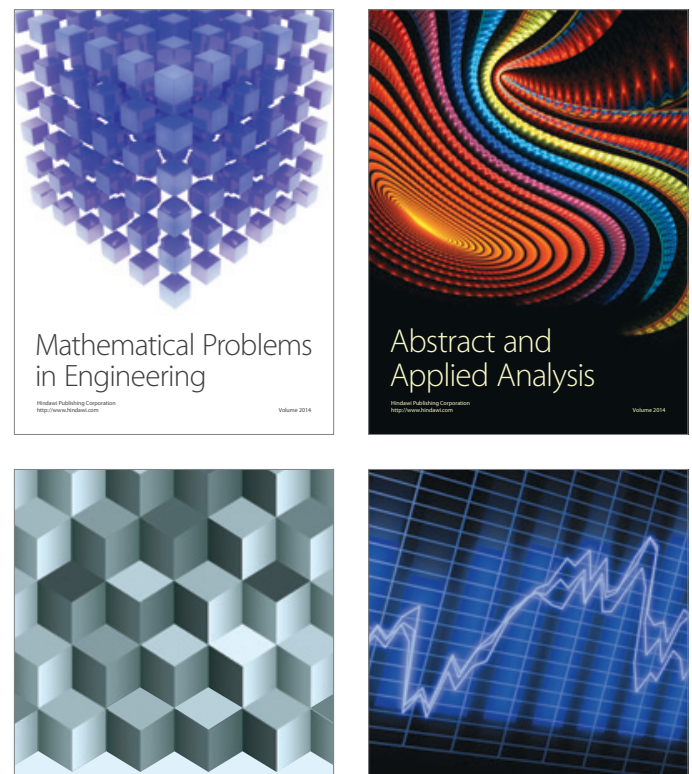

Journal of

Function Spaces

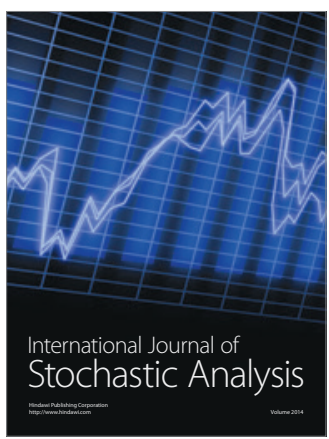

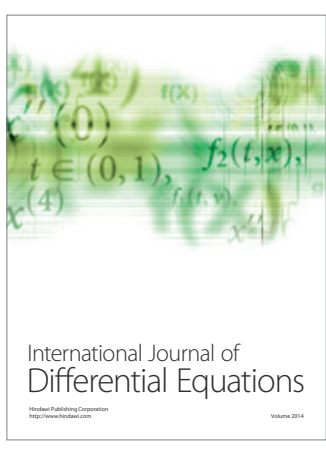
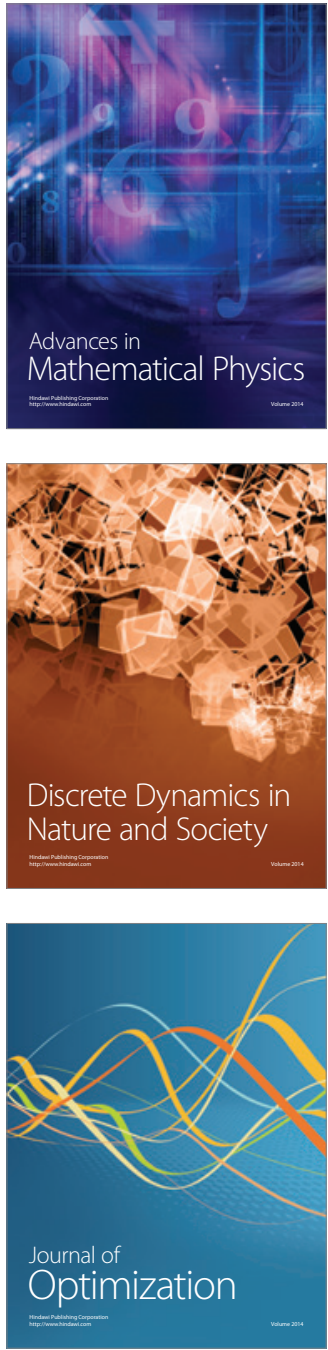